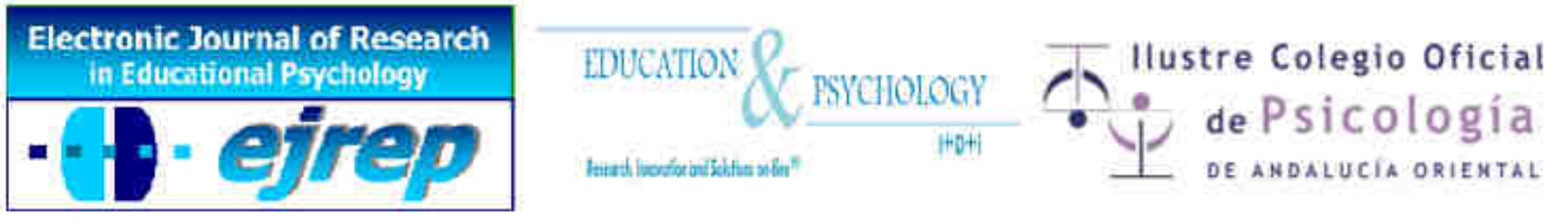

\title{
Satisfacción académica en la universidad: relaciones entre inteligencia emocional y engagement académico
}

\author{
Itziar Urquijo', Natalio Extremera ${ }^{2}$
}

${ }^{1}$ Facultad de Psicología y Educación, Universidad de Deusto, Bilbao

${ }^{2}$ Facultad de Psicología, Universidad de Málaga, Málaga

\section{España}

Correspondencia: Itziar Urquijo . Universidad de Deusto, Facultad de Psicología y Educación. Avenida de las Universidades 24, 48007, Bilbao. España. E-mail: Itziar.urkijo@deusto.es

(C) Universidad de Almería and Ilustre Colegio Oficial de la Psicología de Andalucía Oriental (Spain) 


\section{Resumen}

Introducción. El presente estudio examinó el efecto mediador del engagement académico en la relación entre inteligencia emocional y satisfacción académica controlando variables sociodemográficas y otros constructos clásicos como el rasgo de personalidad de conciencia.

Método. La muestra estuvo compuesta por 203 estudiantes (140 mujeres, 63 hombres) de la Universidad de Deusto con edades comprendidas entre 18 y 27 años. Para el análisis de datos se realizó el análisis bootstrapping para corroborar la existencia de los efectos múltiples de mediación.

Resultados. Los resultados obtenidos indicaron que la inteligencia emocional se asocia significativamente a la satisfacción académica de los estudiantes universitarios. Además, el modelo final mostró una mediación completa del engagement académico en la relación entre inteligencia emocional y satisfacción académica, incluso una vez controladas tanto las variables sociodemográficas como de personalidad.

Discusión y conclusiones. El presente estudio supone uno de los primeros trabajos desarrollados en una muestra de estudiantes universitarios en los que se analiza el efecto mediador del engagement académico en la relación entre inteligencia emocional y satisfacción académica controlando variables sociodemográficas y de rasgos de la personalidad. Así, los hallazgos del presente estudio sugirieron un proceso subyacente en la relación entre inteligencia emocional y la satisfacción académica. Finalmente, se discuten las limitaciones e implicaciones de los resultados obtenidos.

Palabras clave: Inteligencia emocional, satisfacción académica, engagement académico, conciencia, estudiantes universitarios 


\section{Abstract}

Introduction. The present study examined the mediating role of academic engagement in the relationship between emotional intelligence and academic satisfaction when controlling for sociodemographic variables and other classic contructs as conscientiousness personality trait.

Method. The sample included 203 university students (140 women, 63 men) from the University of Deusto with ages ranging from 18 to 27 years. For the analysis, bootstrap methods were used to explore the multiple mediator effects

Results. Results of the study revealed that emotional intelligence was positively associated with academic satisfaction in the sample of university students. Moreover, the final model showed a complete mediation of academic engagement in the relationship between emotional intelligence and academic satisfaction even controlling for sociodemographic and personality traits variables.

Conclusion. . This study represents one of the first attempts to investigate the mediator effect of academic engagement in the relationship between emotional intelligence and academic satisfaction controlling for sociodemographic variables and conscientiousness in a sample of university students. Thus, our findings suggest an underlying process in the relationship between emotional intelligence and academic satisfaction. Finally, limitations of the results and implications for future research are discussed.

Keywords: Emotional intelligence, academic satisfaction, academic engagement, conscientiousness, university students. 


\section{Introducción}

El grado de satisfacción en los estudiantes universitarios ha despertado un creciente interés desde que la UNESCO en su Declaración Mundial sobre la Educación Superior en el Siglo XXI, apuntase al estudiante y sus necesidades como eje central del proceso de calidad educativa (UNESCO, 1998). Esto ha derivado en múltiples investigaciones que han centrado su atención tanto en la satisfacción vital (Huo y Kong, F., 2014; Jonker, Koekemoer y Nel, 2015) como en la satisfacción laboral (Lent y Brown, 2006). A pesar de ello, la importancia de la satisfacción laboral se ha visto reducida debido a la falta de cercanía del estudiante con el mundo laboral, siendo la satisfacción académica la que ocupa un lugar de mayor relevancia (Lent, Singley, Sheu, Schmidt y Schmidt, 2007).

Además, diversos estudios han reforzado el valor positivo de la satisfacción académica definiendo esta variable como factor facilitador del proceso de enseñanza-aprendizaje y posterior desempeño académico y laboral (De Vos \& Soens, 2008; Ojeda, Flores y Navarro, 2011; Park, 2011). Entre los diferentes aspectos que se asocian a la satisfacción académica cabe destacar la importancia del componente personal del alumnado (González, 2014). De este modo, la inteligencia emocional se sitúa entre los factores más relevantes y fundamentales siendo predictor de una mayor satisfacción en el ámbito académico y de una reducción del abandono de los estudios (Salanova, Martínez, Bresó, Llorens y Grau, 2005).

\section{Inteligencia emocional y satisfacción académica}

La inteligencia emocional ha sido objeto de gran debate en investigación debido a la controversia creada en torno a la conceptualización y medición del término (Mayer, Roberts y Barsade, 2008). Así, en la actualidad el constructo ha sido enfocado desde modelos diferentes, presentando aproximaciones al concepto de lo más diversas (Bar-On, 1997; Salovey y Mayer, 1990; Petrides y Furnham, 2003). Uno de los modelos más aceptados en la comunidad científica ha sido el de Mayer y Salovey (1997) que definen la inteligencia emocional como una habilidad para percibir, asimilar, comprender y regular las emociones propias y las de los demás (Mayer y Salovey, 1997). 
En el ámbito de la enseñanza superior, la inteligencia emocional ha estado ligada a aspectos tan fundamentales como son el éxito académico (Saklofske, Austin, Mastoras, Beaton y Osborne, 2012), las relaciones sociales (Lopes, Salovey y Straus, 2003; MartinRaugh, Kell y Motowidlo, 2016), la fatiga en el estudio (Brown y Shutte, 2006) o el bienestar subjetivo entre otros (Kong, Zhao y You, 2012). A pesar de que la relación entre la inteligencia emocional y los mencionados aspectos relativos tanto al bienestar como al éxito académico de los estudiantes universitarios ha sido correctamente establecida, existe la necesidad de ahondar en los sentimientos internos de satisfacción académica para un mejor afrontamiento de los retos académico-profesionales (Boyd, 2002).

La satisfacción académica en la enseñanza superior es comprendida como parte del proceso de la carrera profesional, entendiéndose esta última como la acumulación de las experiencias relacionadas con la carrera (Rothwell y Arnold, 2007). Aunque limitados, existen estudios en los que se demuestra que un mayor nivel de inteligencia emocional se relaciona positivamente con una mejor percepción de satisfacción académica (Holt, 2007; Salanova et al., 2005). A pesar de ello, existe falta de evidencias referente a los potenciales mecanismos que interfieren en esta relación.

\section{Inteligencia emocional, engagement académico y satisfacción académica}

Uno de los posibles mecanismos que podría mediar la relación entre inteligencia emocional y satisfacción académica es el engagement académico. El engagement académico, se define como un estado de bienestar psicológico compuesto por tres dimensiones (vigor, dedicación y absorción) de compromiso intrínseco hacia los estudios (Parra y Pérez, 2010). Por un lado, el vigor es entendido como la voluntad de dedicar el esfuerzo a una acción concreta y persistir ante las dificultades, por otro lado, la dedicación se caracteriza por la involucración en la tarea y la experimentación de orgullo y entusiasmo en la misma y por último la absorción se refiere a la concentración e inmersión en la propia acción. Anteriores estudios han encontrado que el engagement académico produce efectos positivos en diferentes factores académicos como la mejora del rendimiento académico (Kuh, Cruce, Shoup y Kinzie, 2008; Reyes, Brackett, Rivers, White y Salovey, 2012), la acumulación de capital social (Harper, 2008) o la propia adaptación a la universidad (Kuh, Palmer y Kish, 2003). 
Asimismo, diferentes investigaciones han mostrado que aquellos estudiantes que presentan un mayor nivel de engagement académico y, por tanto, están más implicados en su proceso de estudios universitarios, presentan una mayor persistencia durante toda la carrera y posterior graduación (Bridges, Cambridge, Kuh y Leegwater, 2005; Pascarella y Terenzini, 2005; Tinto, 2005).

A su vez, estudios llevados a cabo en estudiantes universitarios, han mostrado la existencia de relaciones significativas entre la inteligencia emocional y el engagement académico incluso cuando se controlan predictores clásicos como el género o la edad (Durán, Extremera, Rey, Fernández-Berrocal y Montalbán, 2006; Extremera, Durán y Rey, 2007). Las personas emocionalmente inteligentes tienen mayor claridad sobre su carrera y manifiestan comportamientos académicos que facilitan tanto la persistencia como el engagement (Drummond, 2014).

A pesar de la importancia de estos resultados, hasta la fecha no existen estudios que examinen el efecto mediador del engagement académico en la relación entre inteligencia emocional y satisfacción académica, controlando ciertos rasgos de personalidad como la conciencia. La conciencia, es entendida como un rasgo de la personalidad dentro del modelo de los cinco grandes que se centra en la diferencia individual en el grado de organización, persistencia y motivación en los comportamientos orientados a objetivos (Costa y Mccrae, 1992). Diversos estudios como el meta-análisis de Poropat (2009) han demostrado que la conciencia tiene efectos comparables con la inteligencia en relación al desempeño académico. En este sentido, un estudiante con un alto nivel de conciencia se compromete con sus estudios y está dispuesto a esforzarse más por alcanzar sus objetivos de la carrera, logrando así una mayor satisfacción académica (McIlveen, Beccaria y Burton, 2013).

\section{Objetivos e hipótesis}

Tomando como base las investigaciones previas, el presente estudio tuvo como principal objetivo analizar el efecto mediador del engagement académico en la relación entre inteligencia emocional y satisfacción académica tras controlar sus niveles previos de conciencia, el género y la edad. En este contexto y a partir de la evidencia empírica previa, se espera que la inteligencia emocional esté relacionada positivamente con la satisfacción académica y que el engagement académico actúe como mediador en la relación entre 
inteligencia emocional y satisfacción académica incluso cuando se controlan otras variables clásicas sociodemográficas y de personalidad.

\section{Método}

\section{Participantes}

La muestra está compuesta por 203 estudiantes de la Universidad de Deusto, España (140 mujeres, 63 hombres). El rango de edad de la muestra oscila entre 18 y 27 años (M= 20.16) y pertenecen a las áreas de Ciencias Sociales y Jurídicas (44.3\%), Ciencias de la Salud (25.6\%) y Artes y Humanidades (30,1\%). El alumnado está repartido entre los diferentes cursos existentes en cada titulación siendo de primer curso el 44,3\%, del segundo curso el $7,9 \%$, del tercero el $5,4 \%$ y por último del cuarto curso el $42,4 \%$.

\section{Instrumentos}

Inteligencia emocional. Para evaluar la inteligencia emocional se utilizó la versión castellana del Wong and Emotional Intelligence Scale (WLEIS; Wong y Law, 2002). Esta prueba de autoinforme está compuesta por 16 ítems con una escala tipo Likert de 7 puntos y presenta la posibilidad de evaluar por un lado cuatro sub-dimensiones (percepción interpersonal, percepción intrapersonal, asimilación y regulación emocional) y, por otro lado, un constructo total. En esta ocasión se computó la puntuación total de inteligencia emocional para lograr una mayor concordancia con los objetivos del presente estudio (i.e. "Tengo un buen control de mis propias emociones"). La versión castellana del Wong and Laws Emotional Intelligence Scale mostró una satisfactoria consistencia en el presente estudio con un Alpha de Cronbach de .91, en línea con lo obtenido en estudios anteriores con muestra española (Rey, Extremera y Pena, 2016).

Satisfacción académica. La Academic Mayor Satisfaction Scale (AMMS; Nauta, 2007) fue el instrumento utilizado para medir la satisfacción académica. Esta medida de 6 ítems que utiliza una escala tipo Likert de 5 puntos analiza facetas específicas de la satisfacción de los estudiantes (i.e. "Me siento bien con la carrera que he escogido"). Para el uso del instrumento en población española, se llevó a cabo una traducción y una posterior 
traducción inversa por parte de personas con dominio nativo en ambas lenguas. La fiabilidad de consistencia interna Alfa de Cronbach obtenida en esta muestra fue de .91 consistente con previas investigaciones (McIlveen, Beccaria y Burton, 2013).

Engagement académico. El engagement académico fue evaluado a través de la versión abreviada de la Utrech Work Engagement Scale for Students (UWESS-9; Schaufeli y Bakker, 2003), la Escala de Engagement Académico (UWES-S; Parra y Pérez, 2010). El instrumento cuenta con 9 ítems que miden las dimensiones de vigor (i.e. Mis tareas como estudiante me hacen sentir lleno de energía), dedicación (i.e. Estoy entusiasmado con mi carrera) y absorción (i.e. Estoy inmerso en mis estudios) ante los estudios. Las alternativas de respuesta se presentan en formato Likert de 6 puntos $(0=$ ninguna vez, $6=$ todos los días $)$. El índice de fiabilidad en este estudio fue de .89 en vigor, .91 en dedicación y .81 en absorción.

Conciencia. Para la evaluación de la conciencia se utilizó la versión castellana de la versión reducida del Goldberg's Bipolar Adjetives (Goldberg, 2012, García, Aluja y García, 2004). Este cuestionario cuenta con 25 ítems de pares de adjetivos evaluados mediante una escala Likert de 9 puntos y evalúa los factores de neuroticismo, extraversión, apertura, agradabilidad y conciencia, consistentes con las dimensiones del Big Five (Smith y Snell, 1996). En esta ocasión, se utilizó la sub-dimensión de conciencia, a fin de lograr los objetivos marcados en este estudio. Dicha sub-dimensión presentó un Alfa de Cronbach de .87 en línea con estudios en los que se utilizó únicamente esta sub-dimensión (McIlveen, Beccaria y Burton, 2013).

\section{Procedimiento}

Los estudiantes participaron de manera voluntaria en la cumplimentación del cuestionario online de múltiples secciones que previamente había sido aprobado por la Comisión de Ética de la Universidad de Deusto. La tarea de completar el cuestionario requería de un tiempo aproximado de 20 minutos y se les ofreció la posibilidad de recibir información sobre sus propios perfiles evaluados una vez finalizado el proceso.

\section{Análisis de datos}

Los estadísticos descriptivos (media, desviación típica y correlaciones de Pearson) fueron calculados para las escalas de inteligencia emocional, satisfacción académica, - 560 - $\quad$ Electronic Journal of Research in Educational Psychology, 15(3), 553-573. ISSN: 1696-2095. 2017. no. 43 http://dx.doi.org/10.14204/ejrep.43.16064 
autoeficacia laboral y conciencia. Para analizar cómo afecta la inteligencia emocional a la satisfacción académica a través de la autoeficacia laboral, se utilizó el procedimiento de mediación de Preacher y Hayes (2004). El nivel de significación fue establecido en p<.01 y todos los análisis mediacionales fueron llevados a cabo utilizando la Macro del SPSS facilitada por Preacher y Hayes.

Para evaluar el posible papel mediador del engagement en la relación entre la inteligencia emocional y la satisfacción académica se llevó a cabo un análisis mediacional. Debido al tamaño muestral del presente estudio, se siguieron las indicaciones de MacKinon, Lockwood y Williams (2004) llevándose a cabo el procedimiento bootstrapping no paramétrico con 5000 repeticiones para estimar los intervalos de confianza del $95 \%$ y analizar el efecto indirecto de la inteligencia emocional sobre la satisfacción académica a través de la variable mediadora. Para realizar un análisis mediacional más completo se controló el efecto de dos covariables sociodemográficas clásicas (edad y género).

Con el objetivo de determinar un modelo teórico de mediación, se siguieron los pasos estipulados por Baron y Kenny (1986). En primer lugar, se debe constatar que existe una asociación significativa entre el predictor (inteligencia emocional) y la variable dependiente (satisfacción académica). En segundo lugar, el predictor y el mediador (engagement) deben estar relacionados. A su vez, el mediador debe presentar una relación con la variable dependiente una vez controlado el efecto del predictor. Por último, el efecto del predictor sobre la variable dependiente debe quedar reducido una vez que el efecto del mediador es introducido. Si el mencionado efecto disminuye hasta alcanzar niveles no significativos estaremos ante una mediación total, si por el contrario el nivel sigue siendo significativo, se considerará mediación parcial.

\section{Resultados}

\section{Resultados descriptivos}

En la Tabla 1 se detallan las medias, desviaciones típicas, fiabilidades y correlaciones de las variables estudiadas. Atendiendo a los coeficientes de correlación de Pearson se observó que la inteligencia emocional se relacionaba positivamente y de manera significativa 
con el grado de satisfacción académica, las tres dimensiones de engagement (vigor, dedicación y absorción), la conciencia y la edad. A su vez, una mayor satisfacción académica se asociaba a un mayor vigor, una mayor dedicación y absorción dentro del factor de engagement académico y una mayor conciencia. En cuanto a las mencionadas dimensiones del engagement académico, todas mostraron una significativa y positiva relación entre sí y con la variable conciencia.

Tabla1. Estadísticos descriptivos y correlaciones parciales entre los instrumentos (n=203)

\begin{tabular}{|c|c|c|c|c|c|c|}
\hline & 1 & 2 & 3 & 4 & 5 & 6 \\
\hline \multicolumn{7}{|l|}{ 1.Inteligencia emocional } \\
\hline 2.Satisfacción académica & $.306 * *$ & & & & & \\
\hline 3.Vigor & $.376^{* *}$ & $.484 * *$ & & & & \\
\hline 4.Dedicación & $.363 * *$ & $.789 * *$ & $.682 * *$ & & & \\
\hline 5.Absorción & $.407 * *$ & $.623 * *$ & $.754 * *$ & $.772 * *$ & & \\
\hline 6. Conciencia & $.276 * *$ & $.380 * *$ & $.291 * *$ & $.362 * *$ & $.273 * *$ & \\
\hline M & 5.15 & 4.41 & 3.78 & 4.91 & 4.24 & 6.60 \\
\hline DT & 0.84 & 0.81 & 1.34 & 1.25 & 1.21 & 1.50 \\
\hline A & .91 & .91 & .89 & .91 & .81 & .87 \\
\hline
\end{tabular}

Nota: ** $p<.01$

Inteligencia emocional y satisfacción académica

Tal y como puede observarse en la Tabla 2, el efecto directo de la inteligencia emocional sobre la satisfacción académica no alcanzó niveles significativos mostrando un valor de .01. Por el contrario, cuando se analizó la relación existente entre la inteligencia emocional y el engagement, se obtuvieron valores significativos de $.51 \quad(p<.001)$ para la dimensión de vigor, .42 ( $p<.001)$ para la dimensión de dedicación y .51 $(p<.001)$ para la dimensión de absorción. En cuanto a la tercera condición, tanto la dimensión de vigor como la dimensión de dedicación mostraron una influencia significativa sobre la satisfacción académica con valores de -.10 (p<.05) y .49 ( $p<.001)$ respectivamente. Por último, el efecto indirecto de las tres dimensiones del engagement se encontraba entre -.1120 y -.0092 en el 
caso de vigor, .0935 y .3447 en el caso de dedicación y -.0178 y .1389 en el caso de absorción con un intervalo de confianza del 95\%. Así, no encontrándose el cero dentro del mencionado intervalo de confianza en el caso de la dimensión de vigor y dedicación y habiéndose reducido el efecto del predictor hasta llegar a alcanzar niveles no significativos, se podría concluir un efecto mediador total del engagement en la relación entre inteligencia emocional y satisfacción académica.

Tabla2. Análisis mediacional de los efectos de la inteligencia emocional sobre la satisfacción académica mediante el engagement controlando la conciencia, el sexo y la edad.

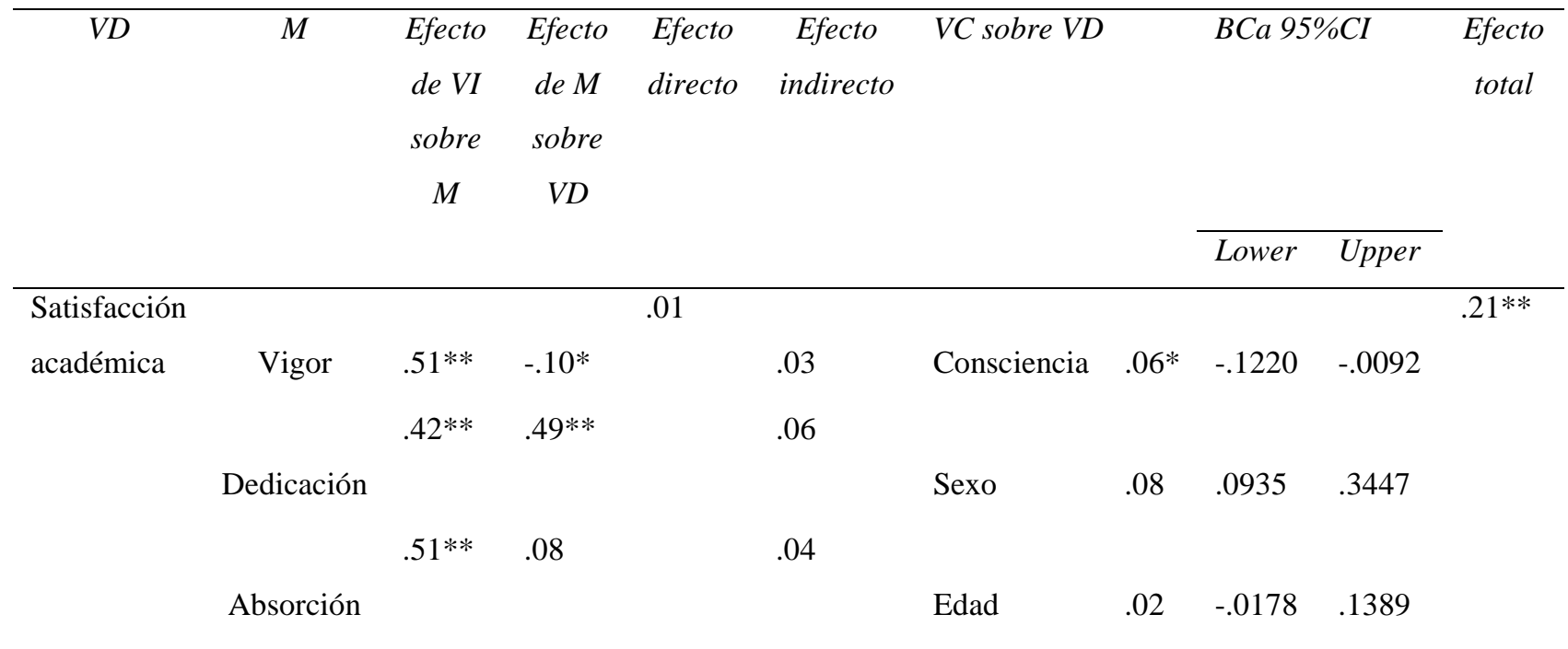

Nota: VD variable dependiente, M mediador, VI variable independiente, VC variable control BCa corrección del sesgo acelerado. ${ }^{*} p<.05 ; * * p<.001$

\section{Discusión}

Esta investigación representa uno de los primeros intentos de examinar el efecto mediador del engagement académico en la relación entre inteligencia emocional y satisfacción académica en estudiantes universitarios. Así, los resultados obtenidos aportan evidencia empírica sobre la importancia que la inteligencia emocional y el engagement académico poseen a la hora de predecir la satisfacción académica. En primer lugar, de manera consistente con estudios previos realizados en la literatura, el presente trabajo encontró que una mayor 
inteligencia emocional por parte de los estudiantes universitarios se relacionaba positivamente tanto con la satisfacción académica como con el engagement académico (Extremera, Durán y Rey, 2007, Trógolo y Medrano, 2012; Oriol-Granado, Mendoza-Lira, Covarrubias-Aplabaza, Molina-López, 2017). A su vez, mayores puntuaciones en engagement académico se asociaron positivamente con mayores niveles de satisfacción académica reforzando hallazgos de previas investigaciones (Wefald y Downey, 2009).

Con respecto a los análisis mediacionales, los resultados indicaron que los niveles de engagement académico mediaron completamente la relación entre inteligencia emocional y satisfacción académica. Así pues, se confirmó que la satisfacción académica de los estudiantes depende del grado de engagement académico, si bien este último se relaciona con la capacidad que tiene la persona para percibir, asimilar, comprender y regular las emociones de uno mismo y de los demás. Estos resultados, podrían apoyar la idea de que aquellos estudiantes universitarios que experimentan un estado agradable de inmersión frente a los estudios y que, a su vez, tienen un compromiso y una dedicación con los mismos, están más satisfechos con su experiencia académica (Caballero, Abello y Palacio, 2007). A su vez, ser capaz de entender los estudios como un reto positivo, disfrutar en las actividades relacionadas y persistir ante los contratiempos y dificultades de los mismos, lleva al estudiante a apreciar una mayor sensación de bienestar y un mejor desempeño y mayor satisfacción en el ámbito académico (Gómez et al, 2015; Wach, Ruffing, Brünken y Spinath, 2016).

Por otro lado, en consonancia con previos estudios, los resultados sugieren que la inteligencia emocional a su vez predice el engagement académico (Durán et al, 2006). La razón de esto, podría ser explicada por la implicación que tienen los procesos emocionales en lo que a vigor, dedicación y absorción académica se refiere. En este sentido, diversas investigaciones indican que aquellos estudiantes con un mayor dominio de sus capacidades emocionales tienen mayor implicación y permanencia en los estudios académicos (Clariana, Cladellas, Badia y Gotzens, 2011; Parker, Hogan, Eastabrook, Oke y Wood, 2006). Además, distintos autores se refieren a la capacidad de manejo de emociones como uno de los mejores predictores del estrés, siendo aquellas personas con mejor manejo de las emociones las que a su vez mejor lidian con las situaciones estresantes (Salovey, Rothman, Detweiler y Steward, 2000; Urquijo, Extremera y Villa, 2016). A esto, cabría añadir que si las emociones no son correctamente percibidas, asimiladas, comprendidas o reguladas se reduce nuestra capacidad 
de manejar las estrategias de afrontamiento activo (Morales, 2017; Salovey, Stroud, Woolery y Epel, 2002). En cuanto a la decisión sobre la carrera, la inteligencia emocional se sitúa como uno de los predictores más relevantes aludiendo a esta capacidad como precursora de la seguridad académica (Di Fabio, Palazzechi, Asulin-Peretz y Gati, 2013). Así, todo ello podría explicar la relación positiva encontrada entre la inteligencia emocional y el engagement académico.

A pesar de las relevantes aportaciones, el estudio no está exento de limitaciones. En primer lugar, los sujetos de la muestra proceden de una misma universidad, siendo una muestra de conveniencia. A fin de poder seguir avanzando en este campo, futuras investigaciones deberían ampliar el origen de la muestra a diferentes universidades de distintas características. En segundo lugar, los datos fueron recogidos utilizando un método online, lo cual podría limitar el control de respuesta. Para evitar las posibles consecuencias negativas, sería conveniente enfocar la recogida hacia una dinámica más presencial. Siguiendo con los datos, la utilización de medidas de autoinforme podría tener sesgos debido a la deseabilidad social. Por último, el uso de un diseño transversal dificulta el hecho de poder mostrar inferencias de causa-efecto en las relaciones. Por tanto, sería recomendable orientar las futuras investigaciones hacia diseños longitudinales y experimentales, utilizando muestra más amplia y con formas de evaluación diferentes tales como medidas de ejecución o formato de entrevistas. A esto, cabe añadir la utilización de distintos análisis estadísticos como pueden ser modelos de ecuaciones estructurales para utilizar un modelo de máxima similitud.

\section{Conclusiones e Implicaciones prácticas}

En cualquier caso, la presente investigación responde a la necesidad de ampliar la investigación del rol de las emociones en las vidas académicas de los estudiantes (Linnenbrink y Pekrun, 2011; Palomera, Fernández-Berrocal y Brackett, 2008; Paoloni, 2014, Thomas, Cassady y Heller, 2017). La realidad de la enseñanza superior ha ido modificándose desde que la Declaración de Bolonia (1999) perfilase unas bases de construcción de un Espacio Europeo de Enseñanza Superior (EEES) basado en una reorganización del conocimiento, una aparición de nuevas expectativas, una internacionalización de la educación y un acercamiento real entre empresa y universidad. Es en esta construcción de una nueva 
visión de la enseñanza superior donde se le otorga especial importancia al hecho de no limitarse a los conocimientos meramente académicos y cognitivos sino desarrollar otras habilidades como la inteligencia emocional para poder hacer frente a nuevos desafíos de adaptabilidad. Hablar de acercamiento real entre universidad y ámbito profesional nos lleva a modificar la enseñanza superior hacia la obtención de habilidades reales para hacer frente a estos nuevos retos. De esta manera, el presente estudio aporta evidencias de la importancia de dotar al estudiante de herramientas imprescindibles para la adaptación tanto al transcurso universitario como a la posterior incorporación profesional clarificando la importancia de la inteligencia emocional y aspectos de la psicología positiva como es el engagement académico en la enseñanza superior.

Por último, dado que diversos estudios argumentan que la inteligencia emocional puede ser modificada a través de programas y experiencias (Dacre- Pool y Qualter, 2012; Di Fabio y Kenny, 2011; Sarrionandia y Garaigordobil, 2017) los resultados hallados en este trabajo refuerzan la idea de considerar las intervenciones en inteligencia emocional con vistas a una mejora del engagement mediante programas que ayuden a fortalecer la satisfacción académica. En conclusión, la implementación de dichos programas, podría tener implicaciones positivas a la hora de disminuir el abandono de los estudios, así como reforzar la motivación e implicación en los mismos, enfocando siempre la enseñanza hacia su proyección de carrera profesional y hacia una mejora del bienestar académico de los estudiantes universitarios.

\section{Referencias}

Avallone, F., Pepe, S. y Porcelli, R. (2007). Autoefficacia percepita nella ricerca del lavoro: scale di misura. En Isfol, Bisogni, valori e autoefficacia nella scelta del lavoro (pp. 133-142). Roma: ISFOL.

Bar-On, R. (1997). The Emotional Quotient Inventory (EQ-i): A test of emotional intelligence. Toronto: Multi-Health Systems, 28.

Boyd, F. B. (2002). Motivation to continue: Enhancing literacy learning for struggling readers and writers. Reading and Writing Quarterly: Overcoming Learning Difficulties, 18 (3), 257-277.Doi: 10.1080/07487630290061818. 
Bridges, B. K., Cambridge, B., Kuh, G. D. y Leegwater, L. H. (2005). Student engagement at minority-serving institutions: Emerging lessons from the BEAMS project. En G. H. Gaither (Eds.). What works: Achieving success in minority retention, (pp. 25- 43). New Directions for Institutional Research. San Francisco: Jossey-Bass.

Brown R.F. y Schutte N.S. (2006) Direct and indirect relationships between emotional intelligence and subjective fatigue in university students. Journal of Psychosomatic Research 60, 585-593. Doi: 10.1016/j.jpsychores.2006.05.001.

Caballero, D., Cecilia, C., Abello, L. L., y Palacio, S. (2007). Relación del burnout y el rendimiento académico con la satisfacción frente a los estudios en estudiantes universitarios. Avances en psicología latinoamericana, 25(2), 98-111.

Clariana, M., Cladellas, R., Badía, M. y Gotzens, C. (2011). La influencia del género en variables de la personalidad que condicionan el aprendizaje: inteligencia emocional y procrastinación académica. Revista Electrónica Interuniversitaria de Formación del Profesorado, 14(3), 87-96.

Costa, P. T. Jr. y McCrae, R. R. (1992c). Revised NEO Personality Inventory (NEO Pl-R) and NEO Five-Factor Inventory (NEOFF/) Professional Manual. Odessa, Florida: Psychological Assessment Resources.

Dacre-Pool, L., \& Qualter, P. (2012). Improving emotional intelligence and emotional selfefficacy through a teaching intervention for university students. Learning and Individual Differences, 22(3), 306-312. Doi: 10.1016/j.lindif.2012.01.010.

De Vos, A., y Soens, N. (2008). Protean attitude and career success: The mediating role of self-management. Journal of Vocational Behavior,73(3), 449-456. Doi: 10.1016/j.jvb.2008.08.007.

Di Fabio, A., \& Kenny, M. E. (2011). Promoting emotional intelligence and career decision making among Italian high school students. Journal of Career Assessment, 19, 21-34. Doi: $10.1177 / 1069072710382530$.

Di Fabio, A., Palazzeschi, L., Asulin-Peretz, L. y Gati, I. (2013). Career indecision versus indecisiveness: Associations with personality traits and emotional intelligence. Journal of Career Assessment, 21, 42-56. Doi: 10.1177/1069072712454698.

Durán, A., Extremera, N., Rey, L., Fernández-Berrocal, P., y Montalbán, M. (2006). Predicting academic burnout and engagement in educational settings: assessing the 
incremental validity of perceived emotional intelligence beyond perceived stress and general self-efficacy. Psicothema, 18(1), 158-164. Doi: 10.1037/t01392-000.

Extremera, N., Durán, A. y Rey, L. (2007). Perceived emotional intelligence and dispositional optimism-pessimism: Analyzing their role in predicting psychological adjustment among adolescents. Personality and Individual Differences, 42(6), 1069-1079. Doi: 10.1016/j.paid.2006.09.014.

Fernández-Berrocal, P., Pérez, J. C., Repetto, E. y Extremera, N. (2004). Una comparación empírica entre cinco medidas breves de inteligencia emocional percibida. En Proceedings of VII European Conference on Psychological Assessment (p. 61). Málaga: European Association of Psychological Assessment.

García, O., Aluja, A. y García, L. F. (2004). Psychometric properties of the Goldberg's 50 personality markers for the Big Five model: a study in Spanish language. European Journal of Psychological Assessment, 20(4), 310-319. Doi:10.1027/1015-5759.20.4.310.

Goldberg, L. R. (1992). The development of markers for the Big-Five factor structure. Psychological assessment, 4(1), 26-42. Doi:10.1037/1040-3590.4.1.26.

Gómez, P., Pérez, C., Parra, P., Ortiz, L., Matus, O., McColl, P. y Meyer, A. (2015). Relación entre el bienestar y el rendimiento académico en alumnos de primer año de medicina. Revista médica de Chile, 143(7), 930-937. Doi: 10.4067/S003498872015000700015 .

González López, I. (2014). Determinación de los elementos que condicionan la calidad de la universidad: Aplicación práctica de un análisis factorial. RELIEVE-Revista Electrónica de Investigación y Evaluación Educativa,9(1), 83-96. Doi: 10.7203/relieve.9.1.4351.

Harper, S. R. (2008). Realizing the intended outcomes of Brown High-achieving African American male undergraduates and social capital. American Behavioral Scientist, 51(7), 1030-1053. Doi: 10.1177/0002764207312004.

Holt, S. (2007). Emotional intelligence and academic achievement in higher education (tesis doctoral). Universidad Pepperdine, Malibu.

Huo, Y. y Kong, F. (2014). Moderating effects of gender and loneliness on the relationship between self-esteem and life satisfaction in Chinese university students. Social indicators research, 118(1), 305-314. Doi: 10.1007/s11205-0130404-x. 
Jonker, C. S., Koekemoer, E. y Nel, J. A. (2015). Exploring a Positive SWB Model in a Sample of University Students in South Africa. Social Indicators Research, 121(3), 815-832. Doi: 10.1007/s11205-014-0658-y.

Kong, F., Zhao, J. y You, X. (2012). Emotional intelligence and life satisfaction in Chinese university students: The mediating role of self-esteem and social support. Personality and Individual Differences, 53(8), 1039-1043. Doi: 10.1016/j.paid.2012.07.032.

Kuh, G. D., Cruce, T. M., Shoup, R., Kinzie, J., y Gonyea, R. M. (2008). Unmasking the effects of student engagement on first-year college grades and persistence. The Journal of Higher Education, 79(5), 540-563. Doi: 10.1353/jhe.0.0019.

Kuh, G. D., Palmer, M., y Kish, K. (2003). The value of educationally purposeful out-ofclass experiences. Involvement in campus activities and retention of first-year college students. En T. L. Skipper y R. Argo (Eds.), Involvement in campus activities and retention of first-year college students (pp. 19-34). Columbia: University of South Carolina, National Resource Center for the First-Year Experience and Students in Transition.

Lent, R. W. y Brown, S. D. (2006). Integrating person and situation perspectives on work satisfaction: A social-cognitive view. Journal of vocational behavior, 69(2), 236247. Doi: 10.1016/j.jvb.2006.02.006.

Lent, R. W., Singley, D., Sheu, H.B., Schmidt, J. A. y Schmidt, L. C. (2007). Relation of social-cognitive factors to academic satisfaction in engineering students. Journal of Career Assessment, 15(1), 87-97. Doi: 10.1177/1069072706294518.

Linnenbrink-Garcia, L. y Pekrun, R. (2011). Students'emotions and academic engagement: introduction to the special issue. Contemporary Educational Psychology, 36(1), 1-3. Doi: 10.1016/j.cedpsych.2010.11.004.

Lopes, P. N., Salovey, P. y Straus, R. (2003). Emotional intelligence, personality and the perceived quality of social relationships. Personality and Individual Differences, 35(1), 641-658. Doi: 10.1016/S0191-8869(02)00242-8.

Marchesi, A. y Martín, A. (1998). Calidad de la enseñanza en los tiempos de cambio. Madrid: Alianza Editorial.

Martin-Raugh, M. P., Kell, H. J. y Motowidlo, S. J. (2016). Prosocial knowledge mediates effects of agreeableness and emotional intelligence on prosocial 
behavior. Personality and Individual Differences, 90, 41-49. Doi: 10.1016/j.paid.2015.10.024.

Mayer, J. D. y Salovey, P. (1997). What is emotional intelligence? En P. Salovey y D. Sluyter (Eds.), Emotional development and emotional intelligence: implications for educators (pp. 3-34). New York: Basic Books.

Mayer, J.D., Roberts, R.D., Barsade, S.G. (2008). Human abilities: emotional intelligence. The Annual Review of Psychology, 59 (1), 507-536. Doi: 10.1146/annurev.psych.59.103006.093646.

McIlveen, P., Beccaria, G., y Burton, L. J. (2013). Beyond conscientiousness: Career optimism and satisfaction with academic major. Journal of Vocational Behavior, 83(3), 229-236. Doi: 10.1016/j.jvb.2013.05.005.

Morales, F. (2017). Relaciones entre afrontamiento del estrés cotidiano, autoconcepto, habilidades sociales e inteligencia emocional. European Journal of Education and Psychology, 10, 41-48. Doi: 10.1016/j.ejeps.2017.04.001.

Nauta, M. M. (2007). Assessing college students' satisfaction with their academic majors. Journal of Career Assessment, 15(4), 446-462. Doi: $10.1177 / 1069072707305762$.

Ojeda, L., Flores, L. Y. y Navarro, R. L. (2011). Social cognitive predictors of Mexican American college students' academic and life satisfaction. Journal of Counseling Psychology, 58(1), 61-71. Doi: 10.1037/a0021687.

Oriol-Granado, X., Mendoza-Lira, M., Covarrubias-Apablaza, C.G. y Molina-López, V.M. (2017). Emociones positivas, apoyo a la autonomía y rendimiento de estudiantes universitarios: el papel mediador del compromiso académico y la autoeficacia. Revista de Psicodidáctica, 22(1), 45-53. Doi: 10.1016/S11361034(17)30043-6.

Palomera, R., Fernández- Berrocal, P. y Brackett, M. (2008). La inteligencia emocional como una competencia básica en la formación inicial de los docentes: algunas evidencias. Electronic Journal of Research in Educational Psychology, 6(15), 437454.

Paoloni, P. V. R. (2014). Emociones en contextos académicos. Perspectivas teóricas e implicaciones para la práctica educativa en la universidad. Electronic Journal of Research in Educational Psychology, 12(3), 567-596.

Doi: http://dx.doi.org/10.14204/ejrep.34.14082

- 570 - Electronic Journal of Research in Educational Psychology, 15(3), 553-573. ISSN: 1696-2095. 2017. no. 43 http://dx.doi.org/10.14204/ejrep.43.16064 
Park, M. (2011). The relationships among learning behaviors, major satisfaction, and study skills of first year medical students. Korean Journal of Medical Education, 23(2), 83-93. Doi: 10.3946/kjme.2011.23.2.83.

Parker, J. D., Hogan, M. J., Eastabrook, J. M., Oke, A. y Wood, L. M. (2006). Emotional intelligence and student retention: Predicting the successful transition from high school to university. Personality and Individual differences, 41(7), 1329-1336. Doi: 10.1016/j.paid.2006.04.022.

Parra, P. y Pérez, C. (2010). Propiedades psicométricas de la escala de compromiso académico, UWES-S (versión abreviada), en estudiantes de psicología. Revista de Educación de Ciencias de la Salud, 7(1), 128-133.

Pascarella, E. T. y Terenzini, P. T. (2005). How college affects students: A Third Decade of Research. San Francisco: John Wiley and Sons.

Petrides, K. V. y Furnham, A. (2003). Technical manual of the trait emotional intelligence questionnaire (TEIQue). London: University of London, Institute of Education.

Poropat, A. E. (2009). A meta-analysis of the five-factor model of personality and academic performance. Psychological bulletin, 135(2), 322- $338 . \quad$ Doi: $10.1037 / \mathrm{a} 0014996$.

Preacher, K. J., y Hayes, A. F. (2004). SPSS and SAS procedures for estimating indirect effects in simple mediation models. Behavior Research Methods, Instruments, \& Computers, 36(4), 717-731.

Rey, L., Extremera, N. y Pena, M. (2016), Emotional competence relating to perceived stress and burnout in Spanish teachers: a mediator model. PeerJ 4:e2087. Doi: 10.7717/peerj.2087.

Reyes, M. R., Brackett, M. A., Rivers, S. E., White, M. y Salovey, P. (2012). Classroom emotional climate, student engagement, and academic achievement. Journal of educational psychology, 104(3), 700-712. Doi: 10.1037/a0027268.

Rothwell, A. y Arnold, J. (2007). Self-perceived employability: Development and validation of a scale. Personnel Review, 36, 23-41. Doi: $10.1108 / 00483480710716704$.

Saklofske, D. H., Austin, E. J., Mastoras, S. M., Beaton, L. y Osborne, S. E. (2012). Relationships of personality, affect, emotional intelligence and coping with student stress and academic success: Different patterns of association for stress and 
success. Learning and Individual Differences, 22(2), 251-257. Doi: 10.1016/j.lindif.2011.02.010.

Salanova, M., Martínez, I., Bresó, E., Llorens, S. y Grau, R. (2005). Bienestar psicológico en estudiantes universitarios: facilitadores y obstaculizadores del desempeño académico. Anales de Psicología, 21(1), 170-180.

Salovey, P. y Mayer, J. D. (1990). Emotional intelligence. Imagination, Cognition, and Personality, 9 (3), 185-211.

Salovey, P., Rothman, A. J., Detweiler, J. B. y Steward, W. T. (2000). Emotional states and physical health. American psychologist, 55(1), 110. Doi: 10.1037//0003O66X.55.1.110.

Salovey, P., Stroud, L. R., Woolery, A., y Epel, E. S. (2002). Perceived emotional intelligence, stress reactivity, and symptom reports: Further explorations using the trait meta-mood scale. Psychology and Health, 17, 611-627. Doi: 10.1080/08870440290025812.

Sarrionandia, A. y Garaigordobil, M. (2017). Efectos de un programa de inteligencia emocional en factores socioemocionales y síntomas psicosomáticos. Revista Latinoamericana de Psicología, 49 (2), 110-118. Doi: 10.1016/j.rlp.2015.12.001.

Schaufeli, W. B., y Bakker, A. B. (2003). The Utrecht Work Engagement Scale (UWES). Test Manual. Utrecht: Department of Social and Organizational Psychology.

Thomas, C.L., Cassady, J.C. y Heller, M.L. (2017). The influence of emotional intelligence, cognitive test anxiety, and coping strategies on undergraduate academic performance. Learning and Individual Differences, 55, 40-48. Doi: 10.1016/j.lindif.2017.03.001.

Tinto, V. (2005). Reflections on retention and persistence: Institutional actions on behalf of student persistence. Studies in Learning, Evaluation, Innovation and Development. 2(3), 89-97.

Trógolo, M. y Medrano, L.A. (2012). Personality traits, difficulties in emotion regulation and academic satisfaction in a sample of argentine college students. International Journal of Psychological Research, 5(2), 30-39.

UNESCO (1998). Higher Education Staff Development: Continuing Mission. Thematic Debate of the Follow-up to the World Conference on Higher Education. Recuperado de http://portal.unesco.org/education/en/ files/12048/10427243910 staff-dev.pdf/staff-dev.pdf. http://dx.doi.org/10.14204/ejrep.43.16064 
Urquijo, I., Extremera, N. y Villa, A. (2016). Emotional Intelligence, Life Satisfaction, and Psychological Well-Being in Graduates: the Mediating Effect of Perceived Stress. Applied Research in Quality of Life, 11(4), 1241-1252. Doi: $10.1007 / \mathrm{s} 11482-015-9432-9$.

Wach, F.-S., Karbach, J., Ruffing, S., Brünken, R., y Spinath, F. M. (2016). University Students' Satisfaction with their Academic Studies: Personality and Motivation Matter. Frontiers in Psychology, 7(55), 1-12. Doi: 10.3389/fpsyg.2016.00055.

Wefald, A. J. y Downey, R. G. (2009). Construct dimensionality of engagement and its relation with satisfaction. The Journal of Psychology, 143(1), 91-112. Doi: 10.3200/JRLP.143.1.91-112.

Wong, C. S., y Law, K. S. (2002). The effects of leader and follower emotional intelligence on performanceand attitude: An exploratory study. The Leadership Quarterly, 13(3), 243-274. Doi: 10.1016/S1048-9843(02)00099-1. 